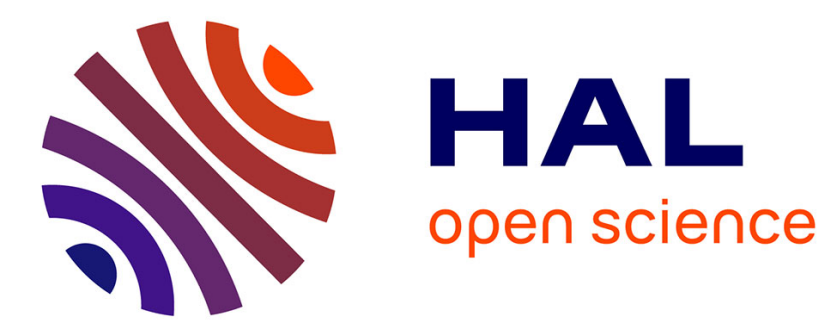

\title{
Implementing an Integer Linear Approach to Multi-objective Phasor Measurement Unit Placement
}

\author{
Amir Baharvandi, Miadreza Shafie-Khah, Saber Talari, João Catalão
}

\section{To cite this version:}

Amir Baharvandi, Miadreza Shafie-Khah, Saber Talari, João Catalão. Implementing an Integer Linear Approach to Multi-objective Phasor Measurement Unit Placement. 8th Doctoral Conference on Computing, Electrical and Industrial Systems (DoCEIS), May 2017, Costa de Caparica, Portugal. pp.297-304, 10.1007/978-3-319-56077-9_29 . hal-01629564

\section{HAL Id: hal-01629564 \\ https://hal.inria.fr/hal-01629564}

Submitted on 6 Nov 2017

HAL is a multi-disciplinary open access archive for the deposit and dissemination of scientific research documents, whether they are published or not. The documents may come from teaching and research institutions in France or abroad, or from public or private research centers.
L'archive ouverte pluridisciplinaire HAL, est destinée au dépôt et à la diffusion de documents scientifiques de niveau recherche, publiés ou non, émanant des établissements d'enseignement et de recherche français ou étrangers, des laboratoires publics ou privés. 


\title{
Implementing an Integer Linear Approach to Multi- Objective Phasor Measurement Unit Placement
}

\author{
Amir Baharvandi ${ }^{1}$, Miadreza Shafie-khah ${ }^{1}$, Saber Talari ${ }^{1}$, and João P.S. Catalão ${ }^{1,2,3}$ \\ ${ }^{1}$ C-MAST, University of Beira Interior, Covilhã 6201-001, Portugal \\ ${ }^{2}$ INESC TEC and the Faculty of Engineering of the University of Porto, Porto 4200-465 \\ ${ }^{3}$ INESC-ID, Instituto Superior Técnico, University of Lisbon, Lisbon 1049-001, Portugal \\ amir.baharvandi@gmail.com; miadreza@ubi.pt; saber.talari@ubi.pt; catalao@fe.up.pt
}

\begin{abstract}
In this paper, an Integer Linear Programming (ILP) problem for a model of Multi-Objective Optimal PMU Placement (MOPP) is proposed. The proposed approach concurrently deals with two objectives. The first objective is the number of phasor measurement units (PMUs) which should be minimized. The second objective function is measurement redundancy which is the number of observable buses in the case of PMU outage. In fact, whatever the amount of second objective increases, the system would be more reliable. Furthermore, some linearized formulations are defined for each nonlinear formula. In fact, the nonlinear nature of formulation related to redundancy is substituted by linear inequality and so there is no nonlinear formula such that the calculation of the problem would be simplified. Finally, a modified 9-bus test system is implemented to show how the proposed method is effective.
\end{abstract}

Keywords: Phasor Measurement Unit (PMU), Multi-objective Mathematical Programming, Integer Linear Programming, Optimal PMU Placement.

\section{Introduction}

The key element of the wide-area monitoring system and application of smart systems is to attain a instantaneous measurement of state variables which Phasor Measurement Units (PMUs) are able to deliver it [1-3]. To increase the security of the system, the future state of the entire network should be estimated [4]. Accordingly, devices known as PMU are used to measure the system's phasors (voltage and current) [5], [6].

In this paper, two previously mentioned objective functions will be described as a Multi-Objective problem in the optimum PMU placement area. Also, to prove the usefulness of the method a case study is tested. 


\section{Relationship to Smart Systems}

Since the metering of the smart systems is of paramount importance to the operator. There are several devices equipping smart cities. These devices are in the kind of electronic devices base of Internet of Things (IoT) [7]. Additionally, the internet facilitates interconnection between people and objects and it is known as the main revolution of the internet infrastructure.

One of these devices using Global Positioning System (GPS) to increase the reliability of the system is Phasor Measurement Unit. It is absolutely certain that implementation of the technological devices has increased drastically in smart cities. In this condition, Phasor Measurement Units can be very applicable in the power electrical systems of smart cities.

To make a clear relationship between the proposed research area and smart system, it should be noted that in the basis of IoT, there is a huge communication between devices using the internet. Likewise, in the smart systems, consumers can manage their consumption in order to decrease their cost of electricity [8].

First relationship between PMU and smart systems is about using the internet. PMUs implement GPS to calculate the voltage phasor of buses and in this process gathers data from buses and uses an internet interconnection with satellite subsequently sends data to a central server. This process is shown in Fig. 1. Another relationship between PMU device and smart system is about managing consumption in smart grids [9].

The calculation of phasors by PMU is utilized to estimate the future states of the power systems and this help to anticipate several parameters such as future consumption in order to supply future loads [10]. State estimating helps the generators and consumers to manage their supply and consumption.

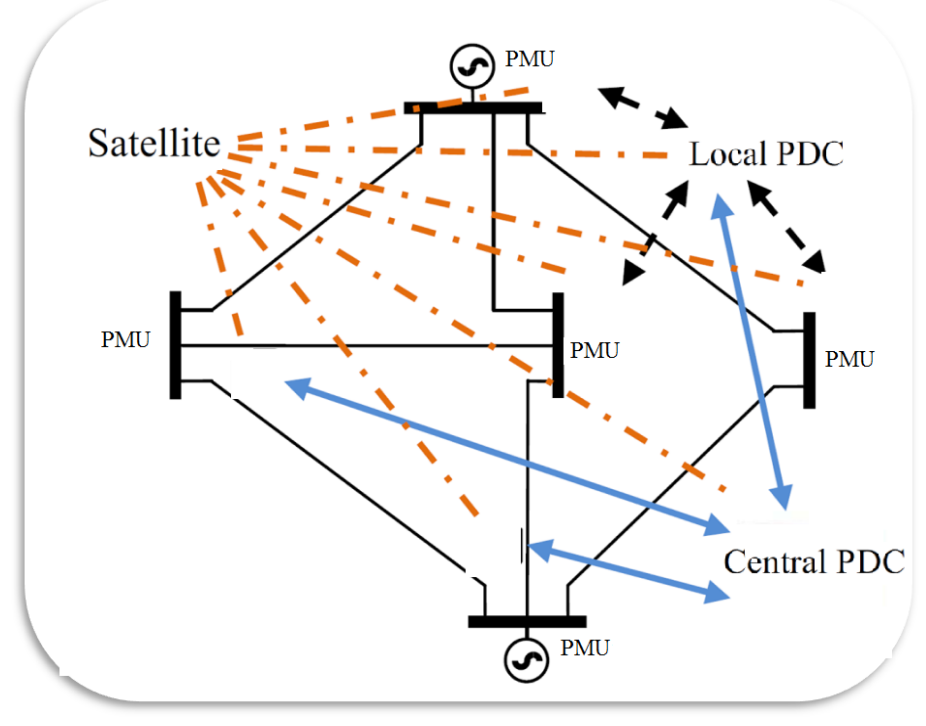

Fig. 1. PMU process 


\section{Problem Formulation}

In the MOPP problem the best condition is making the entire network observable while the redundancy of the system is in a suitable range. It goes without saying that this observability is better to achieve using the minimum PMUs and at the same time all the system constraints would be satisfied.

In the following objective functions and constraints are introduced.

\subsection{Objective Function}

\section{$\mathrm{F}_{1}$ : First Objective Function}

As mentioned before we want to increase the observability of the system (all buses) by minimum cost. Thus, minimum number of PMU which is equivalent the minimum cost is the first objective function:

$$
\min F_{1}=\sum_{\forall i \in I} x_{i}
$$

where $F_{l}$ is the first objective function, $x_{i}$ is a binary variable (which equals to 0 if the bus has not PMU and 1 otherwise) and $I$ is a set for all buses from 1 to $N_{b}$ ( $N_{b}$ is the number of buses).

$F_{2}$ : Second Objective Function

The second objective is redundancy which is associated with contingency occurrence in the system. This contingency may be PMU. It means when a contingency occurs how many buses lose their observability. The desire condition is that we will have the minimum number of unobservable bus. In other word it is absolutely certain that whatever the PMUs increases the number of unobservable buses decreases as well as the redundancy increases. On the contrary, whatever the number of PMUs would be more the cost of installation related to the first objective function increases. So, we have the limitation of PMU and cost and should be aware about it.

Redundancy parameter $(r)$ is equal to the number of observable buses while a contingency occurs. In this study we suppose that this contingency could be PMU loss. The second objective function is introduced as the number of set of buses which are observable when all the contingencies occur separately. This set is defined as $R$ and the member of this set $(r)$ should be maximized. Furthermore, maximizing of $r$ is equivalent to the following formulation:

$$
\min F_{2}=N_{b}-r .
$$

If a PMU is placed on especial bus, that bus and buses connected to that bus are observable. For Example, in Fig. 2, assume that 3 PMUs are located at buses 1, 2, and 7 and all buses are observable. To explain the redundancy criterion, we should disregard PMUs one by one and after each contingency the observability of buses should be analyzed. Now suppose that PMU installed at bus 1 is failed. In this state, 
all the buses are observable too. Accordingly, the PMU located at bus 7 would be failed. In this contingency bus 7 would be unobservable. Similarly, in the case of PMU outage at bus 2, buses 3, 4 and 5 are unobservable. Thus, the number of entire unobservable buses in all PMU outages is 4 and this is the value of second objective function.

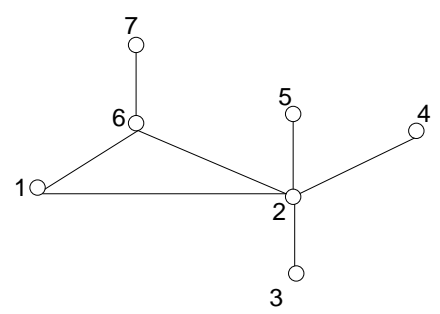

Fig. 2. Example for illustration

\subsection{Constraints}

As mentioned before, an installed PMU results in observability for he installed bus and its adjacent buses. A function $\left(f_{i}\right)$ is introduced as sum of $u_{i}$ for bus $i$ and its neighbors.

$$
f_{i}=G X=\sum_{j \in I} g_{i j} \times u_{j}, \quad \forall i \in I
$$

(3)

where,

$$
\begin{gathered}
\qquad G=\left[g_{i j}\right]_{N_{b} \times N_{b}} \\
g_{i j}= \begin{cases}1, & \text { if bus } i \text { and } j \text { are connected or } i=j \\
0, & \text { otherwise }\end{cases} \\
U=\left[\begin{array}{lll}
u_{1} u_{2} \ldots & u_{N b}
\end{array}\right]^{T}
\end{gathered}
$$

To avoid any unobservable bus, all the functions of $f_{i}$ should be nonzero:

$$
f_{i} \geq 1, \quad \forall i \in I \text {. }
$$

There is another issue related to zero injection buses. These buses may reduce the PMU number using KCL. In fact, when all buses associated with a zero injection bus are observable but one of them is unobservable, the KCL law leads to observability of that unobservable bus. Therefore, $f_{i}$ in (3) would be as follows [11]: 


$$
\begin{aligned}
& f_{i}=\sum_{j \in I} g_{i j} \times u_{j}+\sum_{j \in I} g_{i j} \times t_{j} \times q_{i j}, \quad \forall i \in I . \\
& t_{j}=\sum_{i \in I} u_{i j} \times q_{i j}, \quad \forall j \in I .
\end{aligned}
$$

where, $t_{j}$ is another binary variable ( 1 for zero injection bus and zero otherwise) and $q_{i j}$ represents an auxiliary binary variable. Suppose a zero injection bus $j$ with $t_{j}=1$. In this state in (9) just one of the $q_{i j}$ equals to 1 which means zero injection bus $j$ results in bus $i$ to be observable and the equality (9) would be correct. Hence, the impact of zero injection buses is taken into account as the second part of (8) that is increased if a zero injection bus causes that another bus is observable. The zero injection buses, $t_{j}$ and $q_{i j}$ are explained completely in [11]. Similarly, when a power flow is placed on one line, if one of the sending-end or receiving-end buses of that line is observable, there is no need for other end-bus to have PMU for observability and it can be observable due to other bus. Thus, constraint (7) for line $i j$ which is a power flow line is substituted by:

$$
f_{i}+f_{j} \geq 1
$$

To formulate redundancy mathematically the following formula is presented using another binary variable i.e. $\beta_{i}$ :

$$
\frac{1}{2 \times N_{b}}+\frac{f_{i}-3}{N_{b}} \leq \beta_{i} \leq 1+\frac{f_{i}-3}{N_{b}} \text {. }
$$

In inequality (11), when $f_{i}$ is higher than 2 the left hand side of (11) will be between zero and one, and the other side would be greater than one so $\beta_{i}$ will be 1 . Also, it is be equal to 0 if $f_{i}<2$, then $\beta_{i}=0$. Thus, sum of $\beta_{i}$ denotes the redundant buses with $f_{i}>2$.

$$
F_{2}=N_{b}-\sum_{\forall i \in I} \beta_{i}
$$

This is the constraint of redundancy which should be greater than a specific value according to necessity of system operator.

$$
r \geq p_{\min }
$$

where the required minimum value of redundancy is $p_{\min }$.

\subsection{Multi-Objective Solution Methodology}

The MOPP problem is as follows:

$$
\begin{aligned}
& \min \left\{F_{1}(x), F_{2}(x)\right\} \\
& \text { s.t.: } g(x) \leq 0, \quad h(x)=0 .
\end{aligned}
$$




\section{Simulation Results}

A nine-bus test system has been implemented to simulate the proposed multiobjective optimization problem. The proposed approach has been employed through a Pentium IV, 1-GHz Core i5 with 8 GB RAM using mixed integer linear programming solver CPLEX 9.0 in the GAMS [14]. Fig. 3 shows the 9-bus test system. Except buses 1, 2 and 4 all the buses have load and also there are 2 generation units located at buses 1 and 2 . Thus, bus 4 has no load and generator and is considered as a zero injection bus.

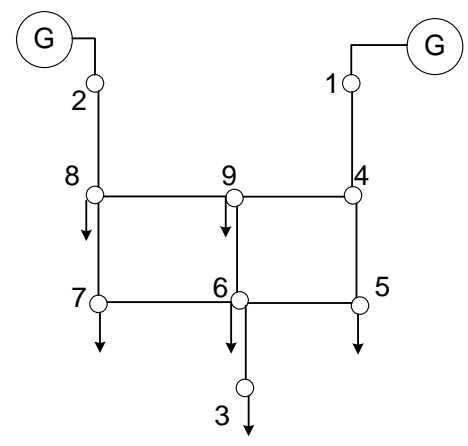

Fig. 3. Modified 9-bus test system

If the first objective function is considered as the main objective, we can have an observable system using lowest number of PMUs that is 3 PMUs at buses 4, 6 and 8 . Subsequently, the amount of second objective stays in the worst state and there is no redundant bus with $f_{i}>2$.

$$
F_{2}=N_{b}-\sum_{\forall i \in I} \beta_{i}=9-0=9
$$

In the flip side, if we solve the single objective problem so that the second objective function is the main objective, there would be 6 PMU at buses 4-9. In this condition the second objective is equal to 3 . The amount of $F_{2}$ cannot be less than 3 , because there are 3 buses in the system which cannot have $f_{i}>2$.

The Pareto points of the proposed Multi-objective method are shown in Fig. 4 and Table I. As we can see from these Figure and Table, two objective functions have conflict manner and they cannot be optimum simultaneously. Whatever the first objective function would be minimized the second one would be worse and conversely. 


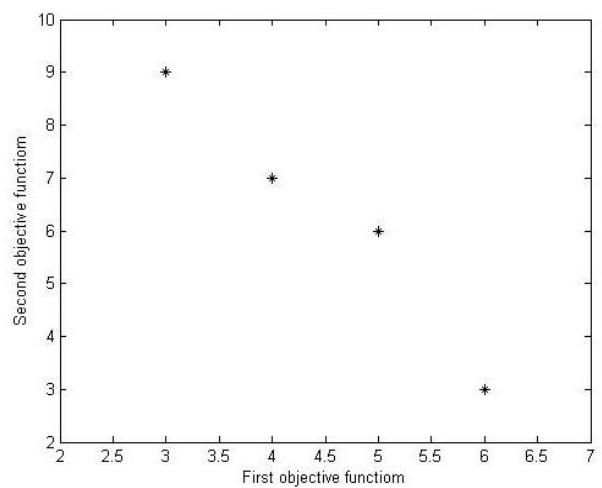

Fig. 4. Pareto set related to test system

Table.1 Pareto points for 9-Bus Test System According to MOPP

\begin{tabular}{l|l|l|l|l}
\hline Pareto \# & $F_{1}$ & $F_{2}$ & $\begin{array}{l}\text { Number of PMU } \\
\text { buses }\end{array}$ & Number of Redundancy buses \\
\hline 1 & 3 & 9 & $4,6,8$ & - \\
\hline 2 & 4 & 7 & $6,7,8,9$ & 7,8 \\
\hline 3 & 5 & 6 & $2,5,6,7,8$ & $6,7,8$ \\
\hline 4 & 6 & 3 & $4,5,6,7,8,9$ & $4,5,6,7,8,9$ \\
\hline
\end{tabular}

Also, constraint (13) can decrease the number of points. For example in the case of $p_{\min }=1$ (the system needs 1 redundant bus) the first row of Pareto set in Table I should be disregarded. It is noteworthy that, the proposed approach can be implemented on larger case studies such as 118-bus or more because the formulation is linear and not complex.

\section{Conclusion}

This paper proposed a multi-objective PMU placement taking into account the redundancy as an extra objective. The redundancy criterion was introduced when a PMU was not in a suitable operation mode. Moreover, the formulation is linear for redundancy which decreases the complexity of the calculation. Two conflict objective functions are minimized simultaneously and the system operator can make a trade-off between points in the Pareto set and choose the best solution point according to the system requirements.

Acknowledgment. This work was supported by FEDER funds through COMPETE 2020 and by Portuguese funds through FCT, under Projects SAICT-PAC/0004/2015 - 
POCI-01-0145-FEDER-016434,

POCI-01-0145-FEDER-006961, UID/EEA/50014/2013, UID/CEC/50021/2013, and UID/EMS/00151/2013. Also, the research leading to these results has received funding from the EU Seventh Framework Programme FP7/2007-2013 under grant agreement no. 309048

\section{References}

1. K. Gharani Khajeh; E. Bashar; A. Mahboub Rad; G. B. Gharehpetian, "Integrated Model Considering Effects of Zero Injection Buses and Conventional Measurements on Optimal PMU Placement," IEEE Transactions on Smart Grid, in press, doi: 10.1109/TSG.2015.2461558.

2. N. Manousakis; G. Korres, "Optimal Allocation of PMUs in the Presence of Conventional Measurements Considering Contingencies," IEEE Transactions on Power Delivery, in press, doi: 10.1109/TPWRD.2016.2524658

3. G. N. Korres, N. M. Manousakis, T. C. Xygkis and J. Löfberg, "Optimal phasor measurement unit placement for numerical observability in the presence of conventional measurements using semi-definite programming," IET Generation, Transmission \& Distribution, vol. 9, no. 15, pp. 2427-2436, 11192015.

4. Manousakis, N. M., Korres, G. N., and Georgilakis, P. S., "Taxonomy of PMU placement methodologies," IEEE Trans. Power Syst., vol. 27, no. 2, pp. 1070-1077, May 2012.

5. Abiri, E., Rashidi, F., and Niknam, T., "An optimal PMU placement method for power system observability under various contingencies," International Transactions on Electrical Energy Systems, vol. 25, no. 4, pp. 589-606, April 2015.

6. Korres, G. N. and Manousakis, N. M., "State estimation and observability analysis for phasor measurement unit measured systems," IET Generation, Transmission \& Distribution, vol. 6 , pp. 902-913, 2012.

7. H. Arasteh et al., "Iot-based smart cities: A survey," 2016 IEEE 16th International Conference on Environment and Electrical Engineering (EEEIC), Florence, 2016, pp. 1-6, doi: 10.1109/EEEIC.2016.7555867

8. Kianoosh G. Boroojeni, M. H. Amini, S. Bahrami, S.S. Iyengar, Arif I. Sarwat, and O. Karabasoglu, "A novel multi-time- scale modeling for electric power demand forecasting: From short-term to medium-term horizon," Electric Power Systems Research, vol. 142, pp. 58-73, January 2017.

9. K. G. Boroojeni, M. H. Amini, and S.S Iyengar, "Overview of the Security and Privacy Issues in Smart Grids," Smart Grids: Security and Privacy Issues. Springer International Publishing, 2017, pp. 1-16.

10.Shahab Bahrami, and Aras Sheikhi, "From demand response in smart grid toward integrated demand response in smart energy hub," IEEE Transactions on Smart Grid 7.2 (2016): 650658 .

11.Aminifar, F., Khodaei, A., Fotuhi-Firuzabad, M., and Shahidehpour, M., "Contingencyconstrained PMU placement in power networks," IEEE Trans. Power Syst., vol. 25, no. 1, pp. 516-523, Feb. 2010.

12.Xu, B., and Abur, A., "Observability analysis and measurement placement for systems with PMUs," in IEEE Power Syst. Conf. Expo., vol. 2, pp. 943-946, Oct. 2004.

13.Chen, J., and Abur, A., "Placement of PMUs to enable bad data detection in state estimation," IEEE Trans. Power Syst, vol. 21, no. 4, pp. 1608-1615, Nov. 2006.

14.Generalized Algebraic Modeling Systems (GAMS), [Online] Available: http://www.gams.com 